\title{
Claves de educación en asma: casos clínicos interactivos
}

\author{
M. Asensi Monzóa ${ }^{a}$ CM. Sánchez Medina ${ }^{b}$ \\ apediatra. CS Serrería. Valencia. España. \\ bPediatra. CS Albalat de la Ribera. Valencia. España.
}

\section{Resumen}

El asma es una enfermedad crónica compleja, con una gran variabilidad y que tiene un enorme impacto, no solo en los pacientes afectados y sus familias, sino también en la sociedad en general.

La prevalencia de asma en la infancia es elevada, afecta alrededor del 10\% de la población infantil y adolescente, siendo en nuestro medio la patología crónica que consume un mayor número de recursos y tiempo en la Atención Primaria pediátrica.

El manejo adecuado del asma implica que se realice un diagnóstico correcto, se evalúe la gravedad y se prescriba el tratamiento indicado. Un componente esencial en el tratamiento del asma infantil es la educación terapéutica, en la que el paciente y su familia han de ser formados y educados, con la finalidad de conseguir un buen control de la enfermedad y mejorar la calidad de vida.

Para que los programas educativos sean efectivos, es necesaria una adecuada formación de los profesionales de la salud, siendo importante una buena comunicación y relación entre sanitarios y pacientes para conseguir que estos entiendan la enfermedad y su tratamiento, y mejoren la adherencia.

En este seminario se van a presentar una serie de casos clínicos para desarrollar diversos contenidos diagnósticos, terapéuticos y educativos del asma infantil.

Palabras clave: Asma. Educación. Niño. Adolescente.

\section{Keys of education in asthma: interactive cases report}

\section{Abstract}

Asthma is a chronic complex disease, with a great variability and a big impact, not only in the affected patients and their families, but also in general society.

The prevalence of childhood asthma is high, it affects about $10 \%$ of the children and adolescents, and it is the chronic condition that consumes more resources and time in pediatric primary care in our population.

Maite Asensi Monzó: maite.asensi@gmail.com

Los autores declaran no presentar conflictos de intereses en relación con la preparación y publicación de este artículo. 
The correct asthma management implies that a proper diagnosis is made, that the seriousness of the condition has been evaluated and that the appropriate treatment has been prescribed. Therapeutic education is an essential component in the treatment of childhood asthma in which the patient and his family have to be trained and educated, in order to achieve a good control of the disease and improve the quality of life.

In order that educational programs are effective it is necessary the adequate training of health professionals, being important an adequate communication and relationship between them and the patients so that they understand the disease, its treatment and they improve the adherence to treatment.

This seminar will present a series of clinical cases to approach various diagnostic, therapeutic and educational contents of childhood asthma.

Key words: Asthma. Education. Child. Adolescent.

\section{Introducción}

El pediatra de Atención Primaria (AP) es, por sus características de accesibilidad y de relación con la familia y el niño, el primer escalón en el diagnóstico, el tratamiento y el control del asma.

Las guías de práctica clínica y proto$\operatorname{colos}^{1-9}$ establecen el papel de la educación terapéutica (ET) como el elemento clave para el manejo y control del asma. Los programas educativos son útiles para disminuir la morbimortalidad y la demanda asistencial ${ }^{10-12}$.

Tanto los profesionales sanitarios como los pacientes y sus familias han de ser formados y educados, para conseguir un buen control de la enfermedad y mejorar su calidad de vida ${ }^{13}$.

Se van a abordar distintos aspectos del asma infantil a través de casos clínicos, y se hará especial énfasis en la educación sanitaria.
Educación terapéutica del paciente asmático

La intervención educativa tiene dos grandes objetivos generales: mejorar la calidad de vida del niño-adolescente y de su familia, hasta conseguir que tengan una vida como la de cualquier niño sin asma; y conseguir el autocontrol, bajo la supervisión del pediatra-educador ${ }^{14}$.

Los resultados más evidentes de la ET se obtienen en pacientes con asma moderado-grave, y son más eficaces cuando se realizan poco después del diagnóstico ${ }^{10,15}$.

\section{¿A quién se debe educar?}

La ET del paciente asmático y su familia es un proceso continuo, dinámico y adaptado que va a permitir conseguir cambios en las actitudes y conductas del paciente y su familia ${ }^{13}$.

Para que los programas educativos sean efectivos, es necesaria una ade- 
cuada formación de los Profesionales Sanitarios, siendo importante una buena comunicación y relación con los pacientes para conseguir que entiendan el asma y su tratamiento, y mejoren la adherencia ${ }^{13,16}$.

\section{¿Quién debe educar?}

Todos los profesionales sanitarios encargados de la asistencia a niños con asma deben participar en el proceso educativo. La AP es el nivel de asistencia más adecuado, por ser un entorno de confianza para la familia, de gran accesibilidad y que cumple el criterio de asistencia integral y continuada al niño. Por tanto, los pediatras y enfermeros de AP deben desempeñar un papel fundamental en la $\mathrm{ET}^{16-18}$, aunque existan una serie de dificultades ${ }^{19}$ (tabla 1).

\section{¿En qué consiste la educación} en asma?

Hay que destacar que educar no es informar, la información es necesaria pero no suficiente. Nuestro objetivo es que el niño y su familia adquieran las habilidades y actitudes necesarias para el control del asma ${ }^{19}$.

La ET será escalonada (tabla 2), desde los conocimientos y habilidades básicos hasta el autocontrol, si es posible ${ }^{18-20}$. Además, será continuada, progresiva y adaptada a la capacidad de cada niño y su familia.

Los conocimientos que hay que enseñar para que el paciente y su familia comprendan el diagnóstico de la enfermedad, la necesidad de exploraciones complementarias y las actuaciones terapéuticas son los que se detallan a continuación.

Tabla 1. Dificultades para educar en el asma infantil desde las consultas de Pediatría de Atención Primaria'

1. Dificultades del educador

- Falta de tiempo: las consultas a demanda en ningún caso pueden ser el lugar idóneo para realizar esta actividad

- Falta de conocimientos sobre el óptimo manejo del asma y los beneficios de la educación

- Falta de conocimientos en las técnicas de la educación y comunicación
2. Dificultades por parte del niño y su familia
- Falta de aceptación de la enfermedad
- Rechazo del paciente al sistema
- Falsas expectativas: espera de curas milagrosas
- Mitos e ideas preconcebidas. Miedos
- Bajo nivel sociocultural, etnia o raza que supongan un inadecuado soporte social, pobre calidad de cuidados
- Factores emocionales como ansiedad, depresión, y otros problemas y enfermedades

Fuente: Domínguez Aurrecoechea B. Educación para la salud en el asma infantil. Mesa redonda, $2 .^{\circ}$ Foro de Pediatría de Atención Primaria en Extremadura. Marzo 2001. 
Conocimiento sobre aspectos básicos del asma

- Analizar los conocimientos previos de la familia sobre la enfermedad: ¿hay alguien en la familia con asma? ¿Conoce los inhaladores y las cámaras?
- Reconocer el asma como inflamación crónica de las vías respiratorias, y analizar el concepto de hiperreactividad y broncoconstricción (apoyo visual con modelos o gráfi$\cos )^{21,22}$ (figura 1). Se explicará que el asma es una enfermedad infla-

\begin{tabular}{|c|c|c|c|c|}
\hline & $\begin{array}{l}\text { Conocimiento de } \\
\text { la enfermedad }\end{array}$ & Prevención & $\begin{array}{l}\text { Tratamiento } \\
\text { farmacológico }\end{array}$ & Autocontrol \\
\hline $\begin{array}{l}\text { Primer escalón: } \\
\text { control médico } \\
\text { del asma }\end{array}$ & $\begin{array}{l}\text { Comprensión } \\
\text { del diagnóstico } \\
\text { de asma } \\
\text { Conocimiento } \\
\text { básico del asma } \\
\text { Identificarlo } \\
\text { como enfermedad } \\
\text { crónica susceptible } \\
\text { de ser manejada }\end{array}$ & $\begin{array}{l}\text { Medidas generales } \\
\text { para evitar } \\
\text { desencadenantes } \\
\text { Evitación } \\
\text { del tabaco }\end{array}$ & $\begin{array}{l}\text { Técnica del inhalador } \\
\text { de uso }\end{array}$ & $\begin{array}{l}\text { Reconocimiento } \\
\text { de síntomas }\end{array}$ \\
\hline $\begin{array}{l}\text { Segundo escalón: } \\
\text { control } \\
\text { compartido } \\
\text { del asma: } \\
\text { médico-familia }\end{array}$ & $\begin{array}{l}\text { Conocer la } \\
\text { diferencia entre } \\
\text { broncoconstrición } \\
\text { e inflamación } \\
\text { Reconocer los } \\
\text { síntomas de asma }\end{array}$ & $\begin{array}{l}\text { Identificar } \\
\text { desencadenantes } \\
\text { específicos } \\
\text { Adoptar medidas } \\
\text { de evitación }\end{array}$ & $\begin{array}{l}\text { Técnica de inhalación } \\
\text { Conocer otros } \\
\text { inhaladores } \\
\text { Consensuar itinerarios } \\
\text { terapéuticos } \\
\text { Diferenciar entre } \\
\text { fármacos aliviadores } \\
\text { y controladores }\end{array}$ & $\begin{array}{l}\text { Manejo de FEM en } \\
\text { consulta } \\
\text { Inicio precoz de } \\
\text { tratamiento de crisis } \\
\text { en domicilio } \\
\text { Participación en la } \\
\text { toma de decisiones }\end{array}$ \\
\hline $\begin{array}{l}\text { Tercer escalón: } \\
\text { autocontrol guia- } \\
\text { do y control por } \\
\text { la familia }\end{array}$ & \begin{tabular}{|l} 
Relacionar la \\
fisiopatología del \\
asma con sus \\
signos y síntomas \\
Conocer el \\
pronóstico \\
probable \\
de su asma
\end{tabular} & $\begin{array}{l}\text { Adecuado control } \\
\text { medioambiental }\end{array}$ & $\begin{array}{l}\text { Los anteriores y evitar } \\
\text { el deterioro de la } \\
\text { función pulmonar }\end{array}$ & $\begin{array}{l}\text { Actuación ante la crisis } \\
\text { y valorar la respuesta } \\
\text { al tratamiento según } \\
\text { plan de acción } \\
\text { Manejo de FEM } \\
\text { en domicilio y diario } \\
\text { de síntomas } \\
\text { Toma de decisiones } \\
\text { autónomas incluso } \\
\text { ante situaciones } \\
\text { nuevas e imprevistas } \\
\text { Estilos de vida positivos } \\
\text { respecto al asma }\end{array}$ \\
\hline
\end{tabular}

Fuente: Díaz Vázquez CA. Fases del control-autocontrol del asma. Modificada de Educación sanitaria a padres y niños con asma. FMC. 1999;6:611-23. 
matoria crónica de las vías aéreas, en cuya patogenia intervienen diversas células y mediadores de la inflamación, condicionada en parte por factores genéticos y que cursa con hiperrespuesta bronquial y una obstrucción variable al flujo aéreo, total o parcialmente reversible, ya sea espontáneamente o por la acción farmacológica ${ }^{3}$. Clínicamente, se caracteriza por episodios recurrentes de sibilancias, disnea, tos y opresión torácica.

A nivel práctico, la definición más útil, especialmente en el grupo del lactante y preescolar, es la del III Consenso Internacional Pediátri$\mathrm{CO}^{23}$ : "Sibilancias recurrentes y/o tos persistente en una situación en la que el asma es probable y se han descartado otras enfermedades menos frecuentes".

Es recomendable proporcionar un documento impreso con la información sobre el asma (www.ae pap.org/avalpap/informacion_sa nitaria_asma.pdf)

- Enseñar cuáles son los síntomas de asma: tos, sibilancias ("pitos"), disnea (dificultad para respirar), dolor u opresión torácica.

- Enseñar a identificar las crisis, y a reconocer síntomas de gravedad (disnea intensa, cianosis, dificultad para hablar o caminar...). Saber identificar una crisis de asma es uno de los aspectos más importantes en el manejo del asma. Cuanto

\section{Figura 1. Modelos 21,22}

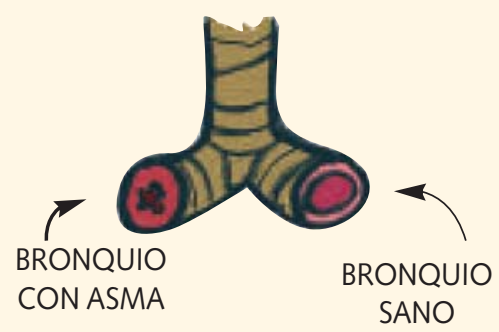

GEMA

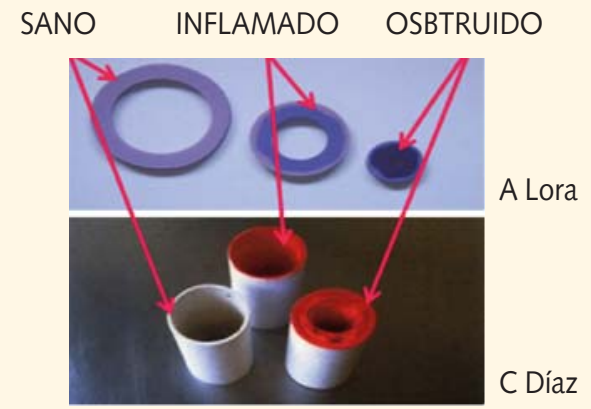


más precozmente se trate una reagudización mejor será el pronóstico de la misma y, probablemente, de la enfermedad en general ${ }^{24}$. Se recomienda dar proporcionar un documento impreso con la información sobre la crisis de asma (www.aepap.org/avalpap/crisis_ asma_hoja1.pdf).

- Enseñar a reconocer los síntomas compatibles con asma por el ejercicio o de esfuerzo. Estos síntomas son aparición de sibilantes, jadeo intenso, cansancio prematuro o tos intensa tras o durante la realización de un ejercicio físico que determine un esfuerzo continuo (correr, subir escaleras, etc.). Aunque la actividad deportiva puede provocar broncoespasmo, no debe contraindicarse y debe ser considerada como altamente recomenda$b^{25}$. Es importante reconocer los síntomas relacionados con el broncoespasmo inducido por el ejercicio sin confundirlos con la baja forma física.

- Saber analizar la coincidencia de la aparición de síntomas con factores desencadenantes (aumento de polinización, cambio de domicilio, contacto con animales, infecciones...).

\section{Conocimientos básicos sobre} prevención

- Explicar qué factores ambientales pueden desencadenar o agravar el asma y cómo evitarlos. La mejor ET es la prevención y, por tanto, enseñar al paciente y a su familia cuáles son los desencadenantes más frecuentes de una crisis para poder evitarlas en la medida de lo posible. Se debe insistir en que la exposición al humo del tabaco siempre empeora los síntomas de asma y se aconsejará evitar la exposición a irritantes ambientales (humos, pinturas, productos de limpieza...). En el caso de que se haya demostrado la implicación de un alérgeno, se deben recomendar las correspondientes medidas de evitación (www.ge masma.com/descargas/GEMA 2003/GEMA\%20pacientes.pdf, www.alergiland.com).

- Explicar medidas preventivas para evitar el asma inducido por ejercicio. Se recomendará evitar ambientes excesivamente fríos y con elevada concentración alergénica, procurando practicar deportes poco asmógenos (natación en espacios abiertos...), realizar un calentamiento previo adecuado, adaptándose al ejercicio progresivamente ${ }^{8,25}$. 
Conocimiento sobre el tratamiento farmacológico

Debemos explicar para qué sirven los broncodilatadores y los antiinflamatorios y cuáles son sus diferencias, así como los posibles efectos secundarios.

\section{Información sobre el tratamiento} farmacológico en la crisis de asma

El objetivo del tratamiento de la crisis de asma es aliviar la obstrucción al flujo aéreo y la hipoxemia tan pronto como sea posible, y planificar la prevención de futuros episodios ${ }^{26}$.

El niño y su familia deben saber para qué sirven los broncodilatadores (de rescate) y conocer sus efectos secundarios. Se informará que son los medicamentos que van a aliviar rápidamente en el momento en que se encuentre mal (con tos y/o con pitos). Hay que explicar el concepto de medicación a demanda o de rescate frente al tratamiento continuado usando, por ejemplo, el modelo de tres tubos ${ }^{22}$.

Los profesionales sanitarios tenemos varias escalas para valorar la gravedad de la crisis de asma. El Pulmonary Sco$\mathrm{re}^{27}$, recomendado en el consenso infantil de asma, es sencillo y aplicable en todas las edades, valora signos clínicos y la saturación de oxígeno.
Además de los signos clínicos, es importante considerar si el paciente ha precisado corticoides orales en crisis previas, el tiempo de evolución de la crisis (cuanto mayor sea el tiempo de evolución, peor será la respuesta al tratamiento), medicación recibida, duración del tratamiento previo con un $\beta_{2}$ adrenérgico de acción corta y valorar si hay antecedentes de riesgo para tener una crisis grave.

En el tratamiento de la crisis de asma se recomienda el uso de agonistas $\beta_{2}$ adrenérgicos inhalados de acción corta de forma precoz y repetida (dosis según la gravedad de la crisis). En las crisis moderadas-graves: asociar oxígeno (para conseguir una saturación $\geq 95 \%$ ), bromuro de ipratropio ${ }^{28}$ y corticoides sistémicos de forma precoz ${ }^{29}$.

Tras la crisis, habrá que revisar el tratamiento preventivo o instaurarlo si no existe, asegurarse de la cumplimentación y de que la técnica de inhalación es correcta, así como repasar los posibles desencadenantes. Es obligatorio revisar el plan de acción; en caso de no tenerlo, es el momento de hacerlo junto con el paciente?

\section{Información sobre el tratamiento farmacológico de mantenimiento}

El niño y su familia han de saber para qué sirven los antiinflamatorios (modificadores) usando el modelo de tres tu- 
bos $^{22}$. Se informará de que se deben tomar a diario, sin interrupción.

Desde el punto de vista de los profesionales sanitarios, según la gravedad del asma se hará un ajuste de la medicación, siguiendo una estrategia de escalonamiento ${ }^{5}$ (tabla 3). Es muy importante insistir en la necesidad de no suspender el tratamiento de mantenimiento, y de actuar precozmente ante una crisis.

\section{Enseñanza en el}

\section{manejo de inhaladores}

En el tratamiento del asma, la vía inhalatoria es la de elección para administrar broncodilatadores y glucocorticoides, con independencia de la edad y de la situación clínica. Mediante esta vía, se consigue una acción más rápida y directa sobre el órgano diana, lo que permite utilizar dosis más bajas de fármaco y disminuye la incidencia de efectos adversos sistémicos.

Se ha demostrado que la ET del niño y su familia en el uso correcto de estos dispositivos puede corregir las técnicas inadecuadas. Por tanto, se deben prescribir los inhaladores después de que el paciente y la familia hayan sido entrenados y comprobemos que realizan la técnica correctamente.

- Es imprescindible que los padres y niños (según la edad) conozcan y dominen el manejo de la técnica inhalatoria.

Tabla 3. Tratamiento de mantenimiento ${ }^{5}$

En pacientes mayores de cinco años de edad, se debe introducir un corticoide inhalado en caso de:

- Exacerbaciones (crisis de asma) en los dos últimos años.

- Empleo de inhaladores beta-2-agonistas tres veces a la semana o más.

- Síntomas tres o más veces a la semana, o un despertar nocturno a la semana.

En menores de cinco años de edad se introducirá el corticoide inhalado en los siguientes casos:

- Lactantes y niños pequeños que han tenido tres o más episodios de sibilancias en el último año, que han tenido más de una noche a la semana con afectación del sueño y que tienen alto riesgo de desarrollar asma persistente por presentar un IPA positivo.

- Lactantes y niños pequeños que de forma constante requieren tratamiento por síntomas de asma más de dos veces por semana.

- Lactantes y niños pequeños con exacerbaciones graves (que requieren beta-2-agonistas de acción rápida inhalado con una frecuencia mayor de cada cuatro horas en 24 horas) que se repiten en menos de seis semanas.

Los pacientes con asma leve intermitente y alta utilización de beta-2-agonistas de acción corta inhalados deben ser revisados en su plan de manejo del asma y se considerará la introducción de medicación preventiva.

IPA: índice predictivo de asma. 
El sistema de inhalación ha de ser elegido en función de la edad, sobre todo en los primeros años (cámaras de inhalación con mascarilla facial o sin ella), y de las preferencias y aptitudes del niño en edades posteriores, de común acuerdo con él y su familia.

Es importante recordar ir modificando el dispositivo con la edad $^{1,13,30:}$ inhalador presurizado con cámara y mascarilla facial en menores de cuatro años, inhalador presurizado con cámara espaciadora con mascarilla facial o sin ella entre los cuatro y los seis años, e inhalador de polvo seco o inhalador presurizado con cámara espaciadora en niños mayores de seis años.

Es recomendable dar la opción de elegir el sistema ya que, en términos educativos, supone un valor añadido al aumentar la confianza, la motivación y la efectividad. Resulta conveniente simplificar al máximo y, si es posible, utilizar el mismo sistema para la medicación antiinflamatoria y la broncodilatadora, con el fin de disminuir al máximo los errores atribuibles a la técnica.

Se debe mostrar la técnica inhalatoria y los diferentes dispositi- vos con envases placebo, enseñándola, comprobando su realización y corrigiendo los posibles errores. Es importante proporcionar un documento impreso, con explicaciones y dibujos de la técnica (www.gemasma.com/des cargas/GEMA2003/GEMA \%20 pacientes.pdf).

- Comprobar la técnica inhalatoria en todas las visitas. La prescripción de cualquier sistema de inhalación debe hacerse solamente después de que el niño y su familia demuestren que realizan la técnica correctamente. Se recomienda reevaluar la técnica inhalatoria en cada visita de control.

\section{Autocontrol del asma}

El autocontrol permite al niño mayor y a la familia tomar decisiones autónomas basadas en la información y educación progresivas.

Para conseguirlo se debe entregar a los pacientes un plan de acción por escrito, personalizado, explicado de forma comprensible, basado en el reconocimiento de síntomas mediante su registro diario (www.aepap.org/avalpap/registro_dia rio_sintomas.pdf) $y$, en casos seleccionados (malos perceptores de síntomas, asma mal controlado, etc.), combinados 
con medición del pico-flujo ${ }^{31}$; con las medidas terapéuticas que deben adoptarse y cuando solicitar ayuda médica ${ }^{11,13,32}$ (www.aepap.org/avalpap/crisis_asma_ hoja1.pdf, www.aepap.org/avalpap/cri sis_asma_hoja2.pdf).

\section{Calidad de vida}

En cada consulta debe valorarse la calidad de vida del paciente y su familia, realizando una serie de preguntas referidas a las últimas cuatro semanas ${ }^{33}$ (tabla 4): ¿ha tosido, ha tenido pitos o le ha costado respirar durante el día o la noche, o al hacer ejercicio o al reír?, ¿ha acudido a Urgencias o ha estado ingresado?
Recordar estimular la práctica de ejercicio físico y recomendar hábitos de vida positivos en relación con el asma.

\section{Conclusiones}

Los profesionales sanitarios, los pacientes y sus familias deben ser educados en asma.

La educación terapéutica debe ser escalonada y adaptada a cada familia.

El plan educativo debe evaluarse de forma periódica para comprobar los resultados obtenidos y valorar las nuevas necesidades en la progresión de conocimientos y habilidades de la familia y el niño.

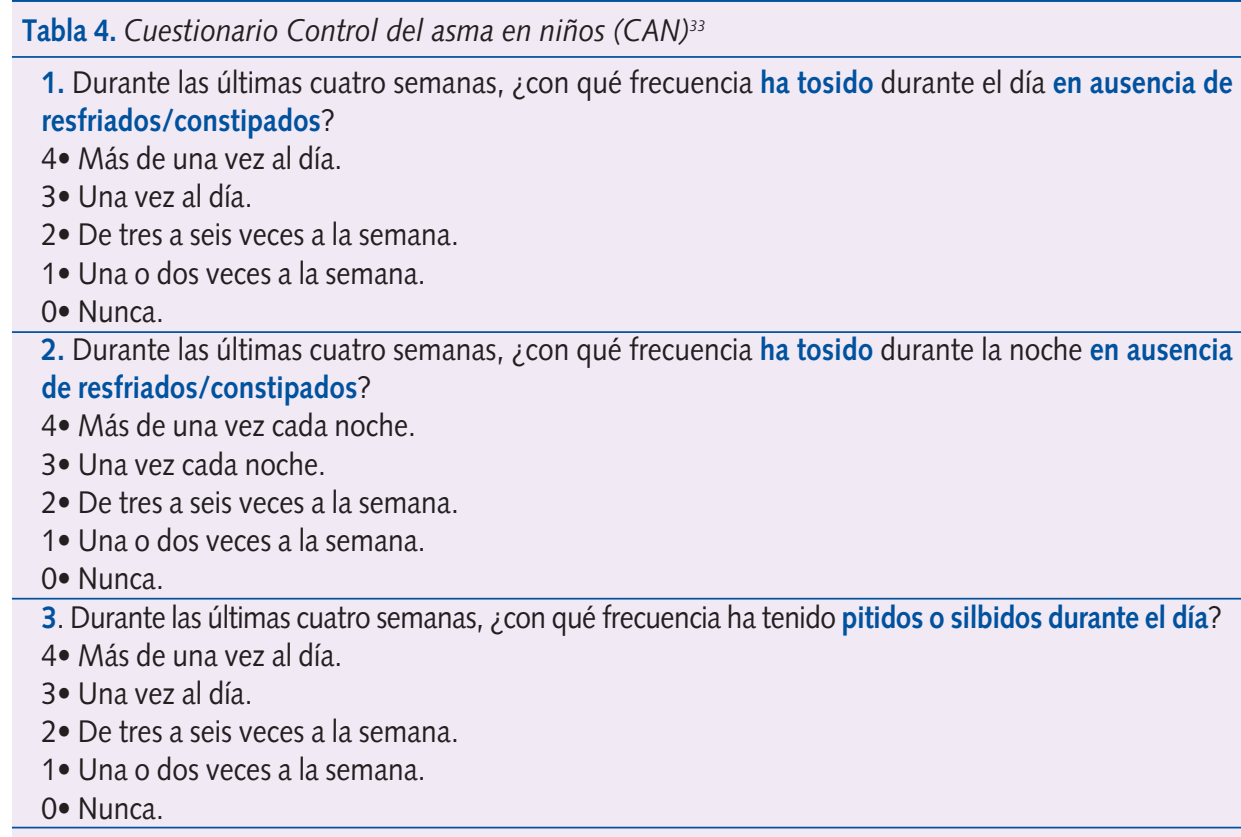


Continúa $\Rightarrow$

4. Durante las últimas cuatro semanas, ¿con qué frecuencia ha tenido pitidos o silbidos durante la noche?

4- Más de una vez cada noche.

3. Una vez cada noche.

2-De tres a seis veces a la semana.

1 - Una o dos veces a la semana.

$0 \bullet$ Nunca.

5. Durante las últimas cuatro semanas, ¿con qué frecuencia le ha costado respirar durante el día? 4• Más de una vez al día.

3• Una vez al día.

2- De tres a seis veces a la semana.

$1 \cdot$ Una o 2 veces a la semana.

$0 \bullet$ Nunca.

6. Durante las últimas cuatro semanas, ¿con qué frecuencia le ha costado respirar durante la noche? 4. Más de una vez cada noche.

3. Una vez cada noche.

2- De tres a seis veces a la semana.

1 - Una ó dos veces a la semana.

$0 \bullet$ Nunca.

7. Cuando el niño hace ejercicio (juega, corre...) o ríe a carcajadas ¿tiene tos o pitos/silbidos?

4• Siempre.

3• Casi siempre.

2- A veces.

$1 \cdot$ Casi nunca.

$0 \bullet$ Nunca.

8. Durante las últimas cuatro semanas, ¿cuántas veces ha tenido que ir a Urgencias debido al asma? 4 Más de tres veces.

3• Tres veces.

2• Dos veces.

1• Una vez.

$0 \bullet$ Ninguna vez.

9. Durante las últimas cuatro semanas, ¿cuántas veces han ingresado en el hospital al niño debido al asma?

4• Más de tres veces.

3• Tres veces.

2・ Dos veces.

$1 \cdot$ Una vez.

$0 \bullet$ Ninguna vez.

Consta de nueve preguntas que se puntúan de 0 a 4. Puntuación máxima 36, mínima 0. Interpretación: 0-7 puntos: el asma está bien controlada; 8-36 puntos: puede que el asma no esté bien controlada. Mayor puntuación implica peor grado de control.

Fuente: Vila JR y cols. Punto de corte que discrimina el nivel de control del asma en el cuestionario del "control del asma en niños" (CAN). An Pediatr (Barc). 2007:66(Supl 2):S76-7. 


\section{Bibliografía}

1. Castillo JA, De Benito J, Escribano A, Fernández $M$, García de la Rubia S, Garde J, y cols. Consenso sobre tratamiento del asma en pediatría. An Pediatr (Barc). 2007;67:253-73.

2. Bacharier LB, Boner $A$, Carlsen $K H$, Eigenmann PA, Fischer T, Götz $M$ et al. The European Paediatric Asthma Group. Diagnóstico y tratamiento del asma en los niños y adolescentes. Informe del Consenso de Practall. Allergy. 2008,63:5-34.

3. Guía española para el manejo del asma (GEMA) 2009. [Consultado el 20/08/2010] Disponible en www.gemasama.com.

4. GINA Report. Global strategy for asthma management and prevention. Global Iniciative for asthma. Updated 2009. [Consultado el 20/08/ 2010]. Disponible en www.ginasthma.org.

5. British Guideline on the management of asthma. A national clinical guideline. British Thoracic Society. Scottish Intercollegiate Guidelines Network. 2008. [Consultado el 25/08/2010]. Disponible en www.brit-thoracic.org.uk.

6. NHLBI. Guidelines for the diagnosis and management of asthma. Summary Report Section 3, Component 2: Education for a Partnership in Asthma Care. (EPR3): 2007. [Consultado el 26/08/2010] Disponible en www.nhlbi.nig. gov/guidelines/asthma/asthgdln.htm.

7. Merino M (Coord.) Guía de práctica clínica sobre asma. Osakidetza/Servicio Vasco de Salud. 2005. [Consultado el 26/08/2010]. Disponible en www.respirar.org/pdf/gpcpv.pdf.

8. Alba Moreno F, Buñuel Álvarez C, Fos Escrivá E, Moreno Galdó $A$, Oms Arias $M$, Puig Congost $M$ y cols. Asma infantil. Barcelona: Institut Catalá de la Salut; 2008. Guies de práctica clínica i material docent, n. ${ }^{\circ}$ 13. [Consultado el 27/08/2010]. Disponible en www.gencat.net/ ics/professionals/guies/asma_infantil.
9. García Merino A, Domínguez Aurrecoechea B. Normas de buena práctica clínica en la atención al niño y adolescente con asma. Documentos técnicos del GVR (publicación DT-GVR-3). [Consultado el 01/09/2010]. Disponible en www.aepap.org/gvr/protocolos.htm.

10. Wolf FM, Guevara JP, Grum CM, Clark NM, Cates CJ. Educational interventions for asthma in children (Cochrane Review). En: The Cochrane Library, Issue 4. Chichester UK: John Wiley \& Sons; 2003.

11. Gibson PG, Powell H. Written action plans for asthma: an evidence-based review of the key components. Thorax. 2004;59:94-9.

12. Gallefoss F, Bakke PS. Impact of patient education and self-management on morbidity in asthmatics and patients with chronic obstructive pulmonary disease. Respir Med. 2000;94:27987.

13. Korta J, Valverde J, Praena M, Figuerola J, Rodríguez CR, Rueda $S$, y cols. La educación terapéutica en el asma. An Pediatr (Barc). 2007; 66:496-517.

14. Korta Murua J. La secuencia educativa. En: Praena Crespo M (ed.). VII Curso de Educadores en Asma. CD-ROM. 1. ${ }^{a}$ edición. Sevilla: Editorial Wanceulen; 2010. p. 17-28.

15. Guevara JP, Wolf FM, Grum CM, Clark NM. Effects of educational interventions for self management of asthma in children and adolescents: Systematic review and meta-analysis. BMJ. 2003;326:1308-9.

16. Figuerola Mulet J. Escenarios de la educación. En: Praena Crespo M (ed.). VII Curso de Educadores en Asma. CD-ROM. 1. a edición. Sevilla: Editorial Wanceulen; 2010. p. 29-37.

17. Moneo Hernández I, Oliván Otal MP, Forés Catalá MD, Lambán Casamayor E. Herramientas en la educación del niño asmático. Rev Pediatr Aten Primaria. 2009;11(Supl 17):s415-22. 
18. Domínguez Aurrecoechea B. Educación del paciente asmático en atención primaria. Bol Pediatr. 2007;47(Supl. 2):88-100.

19. Domínguez Aurrecoechea B. Educación para la salud en el asma infantil. Mesa redonda, 2. ${ }^{\circ}$ foro de Pediatría de Atención Primaria en Extremadura; 2001.

20. Díaz Vázquez CA. Educación sanitaria a padres y niños con asma. FMC. 1999;6:611-23.

21. GEMA. Guía española para el manejo del asma para pacientes, padres y amigos. [Consultado el 01/09/2010]. Disponible en www.ge masma.com/descargas/GEMA2003/GEMA \%20pacientes.pdf.

22. Díaz Vázquez CA. Modelo Tridimensional de los Tres Tubos ${ }^{\odot}$. [Consultado el 01/09/ 2010]. Disponible en www.respirar.org.

23. Warner JO, Naspitz CK. Third Internacional Pediatric Consensus statement on the management of childhood asthma. International Pediatric Asthma Consensus Group. Pediatr Pulmonol. 1998;25:1-17.

24. Jiménez Cortés $A$, Praena Crespo $M$, Lora Espinosa A; Grupo de Vías Respiratorias. Normas de Calidad para el tratamiento de la Crisis de Asma en el Niño y Adolescente. Documentos técnicos del GVR (publicación DT-GVR-1). [Consultado el 02/09/2010]. Disponible en www.aepap. org/gvr/protocolos.htm.

25. Rueda Esteban S. Taller "Enseñando qué es el asma". En: Praena Crespo M (ed.). VII Curso de Educadores en Asma. CD-ROM. 1. a edición. SeviIla: Editorial Wanceulen; 2010. p. 71-83.

26. Callén Blecua $M$, Bamonde Rodríguez $L$. Casos clínicos en asma. En: AEPap. 7. ${ }^{\circ}$ Curso de Actualización Pediatría 2010. Madrid: Exibris Ediciones; 2010. p. 357-65.
27. Smith SR, Baty JD, Hodge D. Validation on the pulmonary score: an asthma severity score for children. Acad Emerg Med. 2002;9:99-104.

28. Plotnick LH, Ducharme FM. Combined inhaled anticholinergics and beta-2-agonists for initial treatment of acute asthma in children (Cochrane Review). En: The Cochrane Library, Issue 4. Chichester, UK: John Wiley \& Sons, Ltd.; 2003.

29. Rowe BH, Spooner C, Ducharme FM, Bretzlaff JA, Bota GW. Early emergency department treatment of acute asthma with systemic corticosteroids (Cochrane Review). En: The Cochrane Library, Issue 4. Oxford: Update Software; 2002.

30. Rueda Esteban S, Valverde Molina, J, López-Seyller M. Taller. Dispositivos para el suministro de la terapia inhalada. En: Praena Crespo M (ed.). VII Curso de Educadores en Asma. CDROM. 1. ${ }^{a}$ edición. Sevilla: Editorial Wanceulen; 2010. p. 111-28.

31. Callén $M$. El flujo espiratorio máximo en el diagnóstico y control del asma. En: Cano A, Díaz CA, Montón JL (eds.). Asma en el niño y adolescente. 2. ${ }^{a}$ ed. Majadahonda (Madrid): Ergon; 2004. p. 59-68.

32. Praena Crespo $M$, Rodríguez FernándezOliva CR, Garrido Halcón D. Taller de Automanejo. Plan de Acción. En: Praena Crespo M (ed.). VII Curso de Educadores en Asma. CD-ROM. 1. ${ }^{a}$ edición. Sevilla: Editorial Wanceulen; 2010. p. 101-10.

33. Vila JR, Cobos N, Pérez-Yarza EG, Garde $J M$, Ibero $M$, Badiola $C$ y cols. Punto de corte que discrimina el nivel de control del asma en el cuestionario del "control del asma en niños" (CAN) An Pediatr (Barc). 2007:66(Supl 2):S76-7. 